\title{
Finite Element Analysis of Flexural Performance of Different Fiber Reinforced Reactive Powder Concrete Beams
}

\author{
Yu QUAN ${ }^{1}$, Changchun PEI ${ }^{1, a,{ }^{*}}$ \\ ${ }^{1}$ Department of Structural Engineering,College of Engineering, YanbianUniversity, Yanji 133002, \\ China \\ a, ${ }^{\star}$ Corresponding author:peicc@yub.edu.cn
}

\begin{abstract}
Keywords: Reactive powder concrete; Finite element analysis; Mid-span bending moment; Mid-span deflection; Ultimate bearing capacity
\end{abstract}

\begin{abstract}
In this paper, the mixing ratio of carbon fiber and steel fiber was changed respectively under different environmental conditions. The influence of different mixing ratio of carbon fiber and steel fiber on the flexural performance of the reactive powder concrete beam was analyzed by finite element method. The results show that: In the condition of corrosive environment, the incorporation of $1.5 \%$ volume fraction of carbon fibers in RPC can significantly improve the mid-span bending moment and deflection of RPC beam in the initial cracking state and the yield state. This can effectively prevent the generation of early cracks, and when the generation of cracks to inhibit the development of cracks. Under normal environmental conditions, the incorporation of 2.0\% volume fraction of steel fibers in RPC significantly increased the mid-span bending moment and deflection in the initial cracking state of the beam, Which can effectively prevent the occurrence of the initial cracks, and effectively delay and inhibit the expansion of cracks after the cracks are generated, greatly improve the brittle performance of RPC and improve the flexural performance of the beam. Based on the results of finite element analysis, the formula for ultimate flexural bearing capacity of fiber reinforced RPC beams is deduced.
\end{abstract}

\section{Introduction}

Reactive powder concrete (RPC) was first developed in France in the 90s of last century, because of its high compressive strength, high tensile strength, low brittleness, excellent durability and other characteristics, RPC has a broad application prospects[1].There are research data that[2 7]: RPC can be incorporated into the fiber to further optimize its performance, making it suitable for a variety of environmental conditions and has the desired technical performance. So the majority of scholars at home and abroad are favored by fiber reinforced RPC, it has a more significant development.However, most researches on fiber reinforced RPC at home and abroad are mainly limited to the experimental study of materials, and few studies have been done on its application in structure.

Therefore, in this paper, the carbon fiber content and steel fiber content of fiber reinforced RPC beam were respectively changed under the condition of corrosive environment and ordinary environment.The finite element analysis is carried out on the mid-span bending moment and deflection under the initial crack state and the yield state of the beam, which can provide references for the engineering application of the fiber reinforced RPC beam.

\section{Test scheme design}

In this paper, the RPC beam without fiber is used as the reference concrete group, in the corrosive environment and ordinary environment, respectively, changes in carbon fiber mixing rate of $0.5 \%, 1.0 \%, 1.5 \%, 2.0 \%$ four levels, changes in the steel fiber mixing rate of $0.5 \%, 1.0 \%, 2.0 \%$, $3.0 \%$ four levels, a total of nine beams were designed. Water-cement ratio was 0.22 , the length of the beam is $1500 \mathrm{~mm}$, the net span is $1200 \mathrm{~mm}$, the beam cross-section dimensions are $150 \mathrm{~mm} \times$ $250 \mathrm{~mm}$ (see Figure 1). Longitudinal tensile reinforcement using 3D25 (HRB500 grade) 
reinforcement,and longitudinal compression steel using 2B10 (HRB335 grade) reinforcement, the stirrups are B10@100 (HRB335 grade) steel bars, the elastic modulus of steel bar is 200GPa. The parameters of the test beam are shown in Table 1.

In order to make the stress state of the simulated beam close to the actual situation, this paper loads two points at the three equal points of the net span of the beam, and replaces the concentrated load with the uniform load of $100 \mathrm{~mm} \times 150 \mathrm{~mm}$.A surface constraint of $100 \mathrm{~mm} \times 150 \mathrm{~mm}$ was applied at a distance of $150 \mathrm{~mm}$ from the beam end to simulate the effect of the rigid plate. The structure of beam specimen is shown in Fig.1.

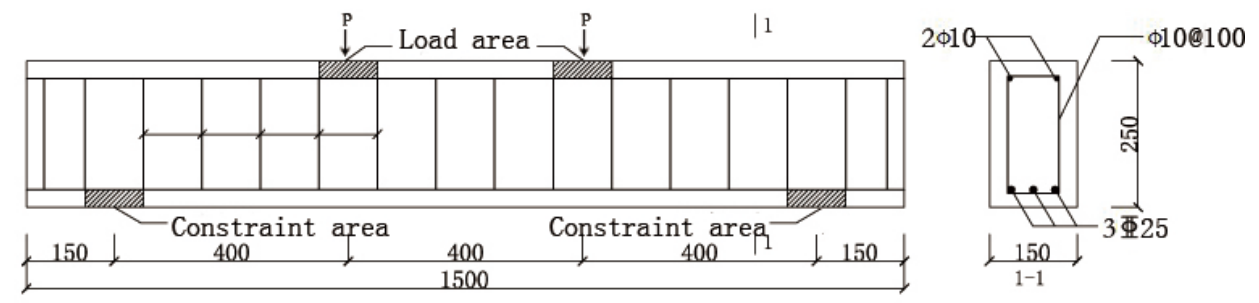

Fig.1 The structure diagram of the specimen (unit: $\mathrm{mm}$ )

Tab. 1 The parameters of the test beam

\begin{tabular}{|c|c|c|c|c|c|c|}
\hline Test number & $\begin{array}{c}\text { Water-cement } \\
\text { ratio }\end{array}$ & $\begin{array}{l}\text { carbon fiber } \\
\text { mixing rate } / \%\end{array}$ & $\begin{aligned} \text { steel fiber } \\
\text { mixing rate } / \%\end{aligned}$ & $\begin{array}{c}\text { cube compressive } \\
\text { strength/Mpa }\end{array}$ & $\begin{array}{c}\text { tensile } \\
\text { strength/Mpa }\end{array}$ & $\mathrm{E} / 10^{4} \mathrm{~N} \cdot \mathrm{mm}^{2}$ \\
\hline Plain & 0.22 & 0 & 0 & 127.3 & 5.5 & 3.896 \\
\hline $\mathrm{C}_{0.5} \mathrm{R}$ & 0.22 & 0.5 & 0 & 130.3 & 7.5 & 3.919 \\
\hline $\mathrm{C}_{1.0} \mathrm{R}$ & 0.22 & 1. 0 & 0 & 138.3 & 7. 9 & 4. 035 \\
\hline $\mathrm{C}_{1.5} \mathrm{R}$ & 0.22 & 1.5 & 0 & 134.2 & 8.1 & 3. 909 \\
\hline $\mathrm{C}_{2.0} \mathrm{R}$ & 0.22 & 2. 0 & 0 & 123.1 & 8.4 & 3.897 \\
\hline $\mathrm{S}_{0.5} \mathrm{R}$ & 0.22 & 0 & 0.5 & 139.9 & 9.2 & 4. 253 \\
\hline $\mathrm{S}_{1.0} \mathrm{R}$ & 0.22 & 0 & 1. 0 & 142.6 & 10.0 & 4.520 \\
\hline $\mathrm{S}_{2.0} \mathrm{R}$ & 0.22 & 0 & 2.0 & 137.8 & 10.5 & 4. 479 \\
\hline $\mathrm{S}_{3.0} \mathrm{R}$ & 0.22 & 0 & 3.0 & 131.6 & 11. 4 & 4. 222 \\
\hline
\end{tabular}

Note: 1) Plain beam: reactive powder concrete beam;

2) $C_{x} R$ : carbon fiber reinforced reactive powder concrete, subscript $x$ value represents the carbon fiber mixing rate;

3) $S_{y} R$ : steel fiber reinforced reactive powder concrete, subscript y value represents the steel fiber mixing rate.

In this paper, RPC beam using a separate model, fiber reinforced RPC selected Solid 65 unit, reinforcement within the beam are used Link8 unit. The modified concrete constitutive relationship method is used to treat the fibers in RPC, the Poisson's ratio for both conventional RPC and steel fiber reinforced RPC is 0.2, and the Poisson ratio for carbon fiber reinforced RPC was 0.24.In the finite element analysis, the reinforcement adopts the bilinear isotropic strengthening model (BISO), and the reinforcement Poisson's ratio is 0.3. The stress-strain relationship of the concrete before cracking and crushing is linear, and the William-Warnke failure criterion is adopted after cracking and crushing.

\section{Finite element results and analysis}

\section{Analysis of flexural behavior of carbon fiber reinforced RPC beam \\ Mid-span moment and deflection of carbon fiber reinforced RPC beam under initial cracking}

Fig. 2 shows the mid-span moment and deflection of the carbon fiber reinforced RPC beam under initial cracking conditions. From Fig. 2 (a), the initial cracking moment of the Plain beam without carbon fiber is $8.4 \mathrm{kN} \cdot \mathrm{m}$, and the initial cracking moment of the RPC beam is obviously enhanced by the incorporation of carbon fiber. With the increase of the carbon fiber content, the bending moment of the beam increases first and then decreases. When the carbon fiber content is $1.5 \%$, the cracking moment reaches the maximum value, which is $60.4 \%$ higher than that of the Plain beam.This is due to the incorporation of carbon fiber, fiber and cementitious materials 

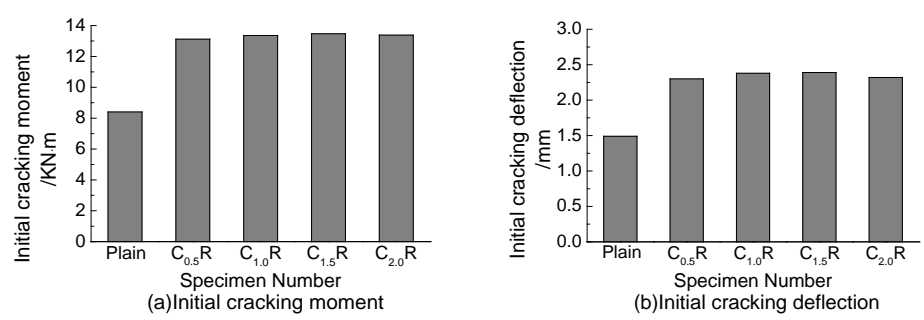

Fig.2 Mid-span moment and deflection of the carbon fiber reinforced RPC beam under initial cracking conditions

together with the force, carbon fiber hinders the expansion of the initial crack, effectively play an early toughening effect.

From Fig.2 (b), we can see that, when carbon fiber is added, the maximum deflection in the initial crack of each group is obviously increased compared with the plain beam.This is because with the increase of the carbon fiber mixing ratio, the toughness of the beam is improved, the beam can bear the larger initial cracking load, delayed the appearance of the initial cracks, and increased the mid-span deflection. With the increase of carbon fiber content, the deflection of crack increases and then decreases. When the content of carbon fiber is $1.5 \%$, the mid-span deflection at the initial crack reaches the maximum, which is $59.2 \%$ higher than that of the plain beams.

\section{Equivalent cracking von Mises stress}

Fig. 3 shows the equivalent cracking von Mises stress of carbon fiber reinforced RPC beams. It can be seen from the figure, in the initial cracking state, the maximum cracking stress of the beam occurs in the compression zone in the middle and upper part of the beam span,at this time,the compressive reinforcement and compressive zone concrete share the compressive stress. With the incorporation of carbon fiber, the maximum crack compressive stress of the beam increases, which is $56.4 \% \sim 60.2 \%$ higher than that of the Plain beam. This is due to the incorporation of carbon fiber, making the carbon fiber and cementitious materials share the stress, constraining the development of cracks, and improving the initial cracking stress of the beam.

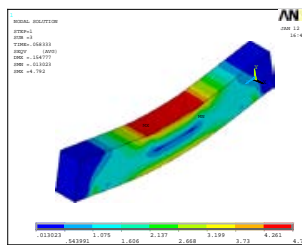

(a) Plain

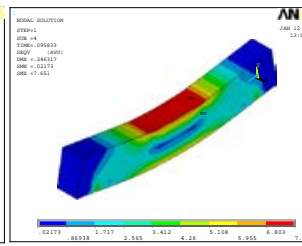

(b) $\mathrm{C}_{0.5} \mathrm{R}$

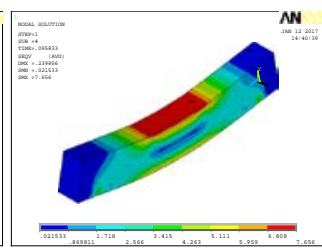

(c) $\mathrm{C}_{1.0} \mathrm{R}$

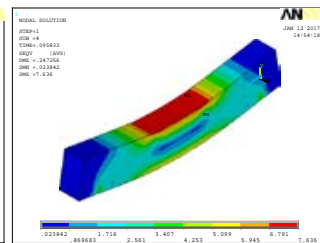

(d) $\mathrm{C}_{1.5} \mathrm{R}$

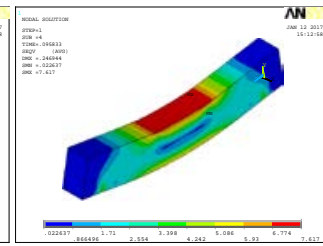

(d) $\mathrm{C}_{2.0} \mathrm{R}$

Fig.3 Equivalent cracking von Mises stress of carbon fiber reinforced RPC beam

\section{Mid-span moment and deflection of carbon fiber reinforced RPC beam under yield condition}

Fig. 4 shows the mid-span bending moment and deflection of the carbon fiber reinforced RPC beam under yield conditions.It can be seen from Fig.4 (a) that when the carbon fiber content is changed from $0.5 \%$ to $1.5 \%$, the yield bending moment of the beam increases with the increase of carbon fiber content, and when the carbon fiber content is $1.5 \%$, the mid-span bending moment reaches the maximum. The results show that the incorporation of carbon fiber effectively enhances the mechanical properties of RPC, especially the tensile strength, which improves the tensile and
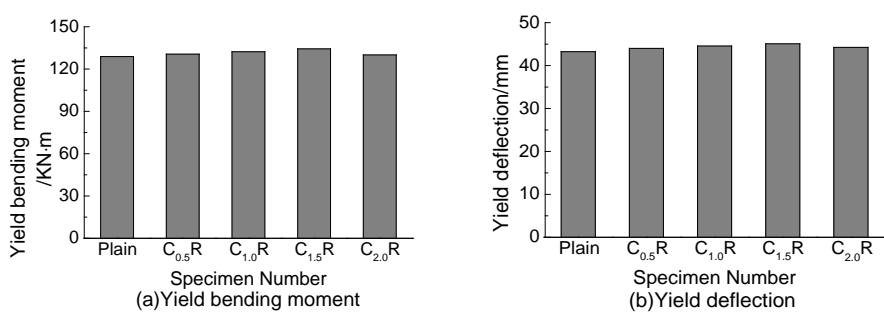

Fig.4 Mid-span moment and deflection of the carbon fiber reinforced RPC beam under yield condition 
compression ratio of RPC beam, and improve the flexural performance of the beam.However, the carbon fiber content increased further and the bending moment decreased, indicating that excessive incorporation of carbon fiber also increased the internal defect of RPC, that is, When the carbon fiber reinforced effect is less than the weakening effect of the defect, the flexural strength of the RPC beams decreases with the increase of the fiber content.

It can be seen from Fig. 4 (b) that, with the increase of the carbon fiber content in the yield state, the mid-span deflection of the beams in each group increased to different extents, which is $1.8 \% \sim 4.3 \%$ higher than that of the Plain beam. When the carbon fiber content is $1.5 \%$, the mid-span deflection of the beam reaches the maximum. This is because when the carbon fiber content is $1.5 \%$, the effect of carbon fiber on the flexural performance of RPC beam is the most obvious. That is, under the same loading mode, the beam with $1.5 \%$ carbon fiber content finally yielded, so the yield deflection value is the largest, which is the same as the yield bending moment of the beam.

\section{Equivalent yield von Mises stress}

Fig. 5 shows the equivalent yield von Mises stress of carbon fiber reinforced RPC beams. It can be seen from the figure that the maximum yield stress of the beam in the yield stage occurs in the upper compression zone. This is because when the beam reaches the yield load, the steel bar exits the work and the stress of the steel bar is transferred to the concrete in the compression area,the compressive stress of concrete increases rapidly, so it is crushed quickly. Compared with the Plain beam, the maximum yield stress of carbon fiber reinforced RPC increases, which is $2.5 \%$ 8.2\% higher than that of Plain beam. This is mainly with the increase of carbon fiber mixing rate, the concrete matrix and fiber together to bear the stress. When the matrix cracks, the fiber across the crack effectively inhibit the development of cracks.And fiber pulled out from the matrix and fibers are pulled off all need to consume a lot of energy, so the flexural performance of beam is improved.

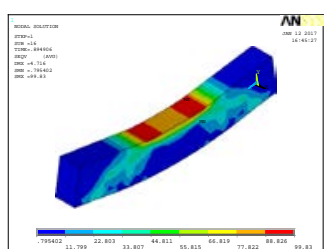

(a) Plain

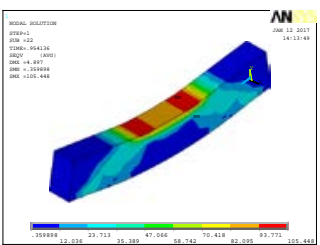

(b) $\mathrm{C}_{0.5} \mathrm{R}$

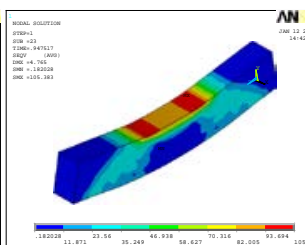

(c) $\mathrm{C}_{1.0} \mathrm{R}$

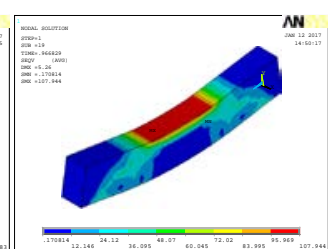

(d) $\mathrm{C}_{1.5} \mathrm{R}$

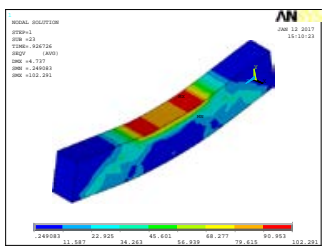

(d) $\mathrm{C}_{2.0} \mathrm{R}$

Fig.5 Equivalent yield von Mises stress of carbon fiber reinforced RPC beam

\section{Analysis of flexural performance of steel fiber reinforced RPC beam}

Mid-span moment and deflection of steel fiber reinforced RPC beam under initial cracking condition

Figure 6 shows the mid-span bending moment and deflection of the steel fiber reinforced RPC beam under initial cracking conditions.From the figure 6 (a) we can see, in the initial cracking stage with the increase of steel fiber content, the mid-span bending moment of each group of beams is significantly increased. When the steel fiber content is $2.0 \%$, the mid-span bending moment of the beam reaches the maximum value. This is due to the fact that the steel fibers are added into the RPC, so that the fibers and the cementitious materials are combined together to bear the stress, which effectively restricts the appearance and development of the initial cracks.

From Fig. 6 (b), it can be seen that the incorporation of steel fibers significantly increases the mid-span deflection of each group of beams under initial cracking conditions compared with the
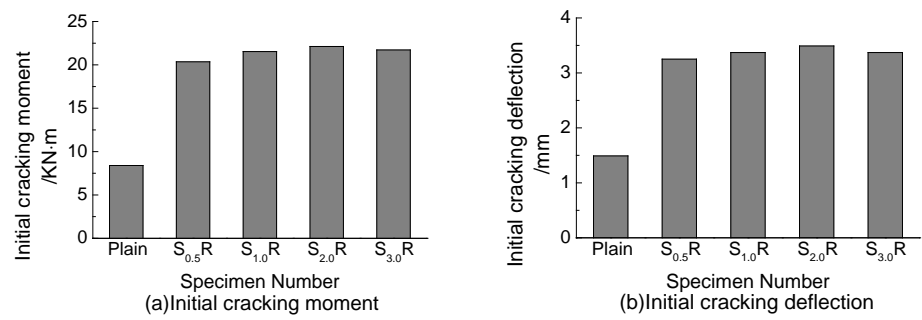

Fig.6 Mid-span moment and deflection of the steel fiber reinforced RPC beam under initial cracking condition 
plain beams.That is, in the initial cracking state, with the increase of steel fiber content, the mid-span deflection of the beam increases first and then decreases. When the steel fiber content is $2.0 \%$, the mid-span deflection of the beam reaches the maximum value, which is $134.2 \%$ higher than that of the Plain beam. This is due to the incorporation of steel fibers, which significantly improves the bending resistance of RPC beams and increases the initial cracking load of the beams. The steel fibers effectively prevent the occurrence of initial cracks, thus increasing the mid-span deflection of the RPC beams during initial cracking.

\section{Equivalent cracking von Mises stress}

Figure 7 shows the equivalent cracking von Mises stress of steel fiber reinforced RPC beams. It can be seen from the figure, in the initial cracking state, the maximum cracking stress of the beam occurs in the compression zone in the middle and upper part of the beam span.It can be seen from the figure, in the initial cracking state, the maximum cracking stress of the beam occurs in the compression area, this is due to stress concentration in the concrete near the loading area.With the increase of the steel fiber content, the maximum cracking stress of each group of beams increased. Compared with the Plain beams, the maximum cracking stress of each group of beams increased by $156.9 \% \sim 163.7 \%$. This is because the incorporation of steel fibers into the RPC effectively inhibits the appearance of the initial cracks, so that fiber and concrete as a whole bear the load, thus significantly increasing the stress of concrete at the initial cracking stage.

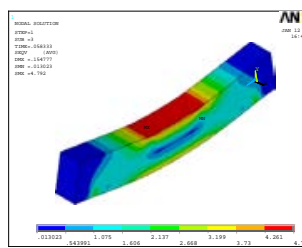

(a) Plain

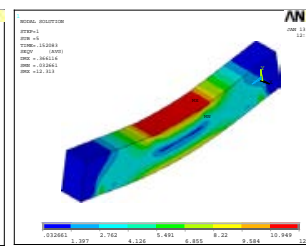

(b) $\mathrm{S}_{0.5} \mathrm{R}$

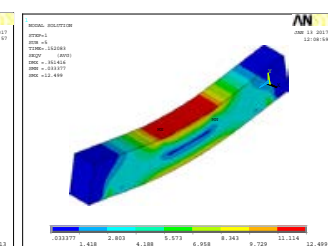

(c) $\mathrm{S}_{1.0} \mathrm{R}$

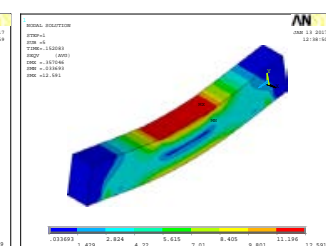

(d) $\mathrm{S}_{2.0} \mathrm{R}$

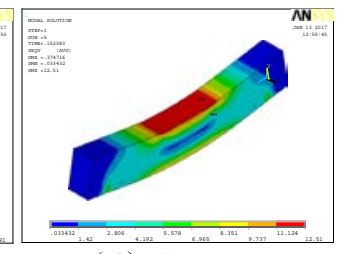

(d) $\mathrm{S}_{3.0} \mathrm{R}$

Fig.7 Equivalent cracking von Mises stress of steel fiber reinforced RPC beam

Mid-span moment and deflection of steel fiber reinforced RPC beam under yield condition.

Fig. 8 shows the mid-span bending moment and deflection of the steel fiber reinforced RPC beam under yield conditions.It can be seen from Figure 8 (a), in the yield state, with the increase of the steel fiber content, the mid-span moment of each group of beam are improved, which is 2.1\% 5.8\% higher than that of the Plain beam. When the steel fiber content is $2.0 \%$, the mid-span bending moment reaches the maximum value, and with the further increase of the steel fiber content, the bending moment decreases slightly. This indicates that there is a critical value of steel fiber mixing rate, and the fiber has the most obvious toughening effect on the RPC beam under the critical mixing ratio, which can obviously improve the flexural performance of the beam.

From the figure 8 (b), we can see that in the yield state, with the increase of the steel fiber mixing rate, the mid-span deflection of each group of beam is increased, which is $4.0 \% \sim 7.2 \%$ higher than that of the Plain beam. When the steel fiber content is $2.0 \%$, the mid-span deflection of the beam reaches the maximum. This is because when the beam cracks, the steel fiber between cracks began to work, the steel fiber is pulled out from the matrix need to consume large amounts of energy, effectively delay and inhibit the expansion of cracks, which greatly improves the brittle performance of RPC and improves the flexural performance of the beam. Therefore, the mid-span deflection of the beam is increased in the yield state.
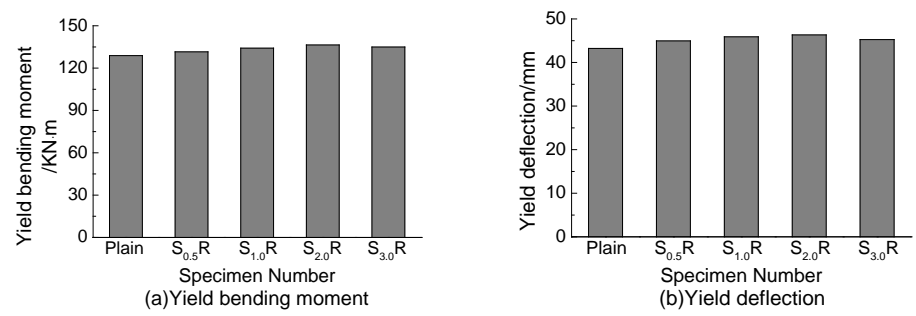

Fig.8 Mid-span moment and deflection of the steel fiber reinforced RPC beam under yield condition 


\section{Equivalent yield von Mises stress.}

Fig. 9 shows the equivalent yield von Mises stress of steel fiber reinforced RPC beams. It can be seen from the figure, the maximum yield stress of the beam in the yield stage occurs near the compression zone in the upper part of the beam. This is mainly because when the beam reaches the yield load, the steel exits the work and the load is borne by the RPC alone. The concrete in the compression area is rapidly crushed due to the rapid growth of stress. With the increase of the steel fiber content, the maximum yield stress of the beam increases to different extents, which is $5.2 \% \sim 14.6 \%$ higher than that of the Plain beam.This is because the incorporation of steel fiber, significantly improved the mechanical properties of RPC, especially the tensile strength. The fiber effectively improves the toughness of the RPC beam and increases the flexural capacity of the beam, so the maximum compressive stress of the concrete is increased under the yield condition.

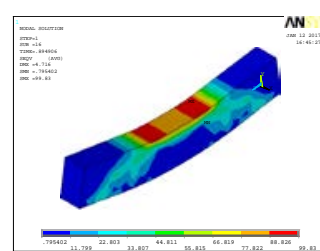

(a) Plain

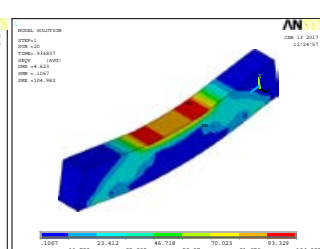

(b) $\mathrm{S}_{0.5} \mathrm{R}$

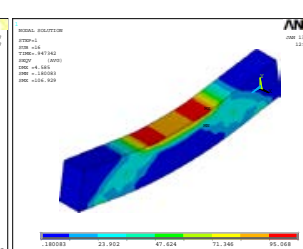

(c) $\mathrm{S}_{1.0} \mathrm{R}$

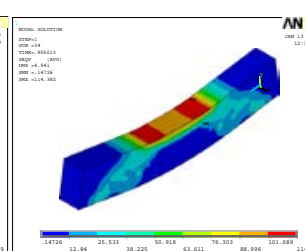

(d) $\mathrm{S}_{2.0} \mathrm{R}$

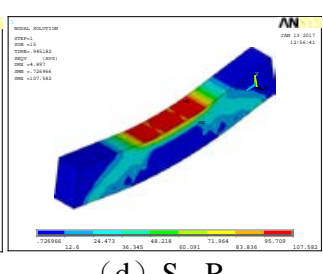

(d) $\mathrm{S}_{3.0} \mathrm{R}$

Fig.9 Equivalent yield von Mises stress of steel fiber reinforced RPC beam

\section{Simulation formula for ultimate bearing capacity of fiber reinforced RPC beam}

\section{Derivation of Simulation Formula for Ultimate Bearing Capacity.}

Because the mechanical properties of RPC are different from those of ordinary high-strength concrete, that is, RPC has higher tensile strength, and the tensile strength is more significantly increased when the fiber is incorporated into RPC.Therefore, it is necessary to consider the effect of RPC in the tensile zone on the flexural bearing capacity when calculating the bearing capacity of the beam section. This paper is based on the formula of ultimate bearing capacity proposed by Wang Zhao-ning [8], the formulas for calculating the ultimate bearing capacity of carbon fiber reinforced RPC beam and steel fiber reinforced RPC beam are deduced respectively. Basic formula:

$$
\begin{aligned}
& M_{u}=0.85 f_{c} b x\left(h_{0}-\frac{x}{2}\right)-f_{t} b x_{t}\left(\frac{x_{t}}{2}-a_{s}\right) \\
& x=\frac{A_{s} f_{y}+f_{t} b h}{b\left(0.85 f_{c}+1.54 f_{t}\right)}
\end{aligned}
$$

In the formula: $M_{u}$ - - Flexural bearing capacity of RPC beam section;

$f_{c}$ - Cubic compressive strength of RPC;

$f_{t}$ - - Tensile strength of PRC;

$f_{y}$ - - Tensile strength of tensile reinforcement;

$A_{s}$ - - Sectional area of tensile reinforcement;

$a_{s}$ - - The distance from the edge of the tension zone to the resultant point of the tensile reinforcement;

$x_{t}, x-$ - Height of tension zone and height of compression zone, $x_{t}=h_{0}-x$;

Considering the enhancement of ultimate bearing capacity of RPC beams after incorporation of carbon fiber and steel fiber, the formula (1) is modified:

$$
\begin{aligned}
& M_{c u}=\left(1+\alpha_{c} \lambda_{c}\right) M_{u} \\
& M_{s u}=\left(1+\alpha_{s} \lambda_{s}\right) M_{u}
\end{aligned}
$$

In the formula: $M_{c u}-$ Flexural bearing capacity of carbon fiber reinforced RPC beams section;

$M_{s u}$ - -Flexural bearing capacity of steel fiber reinforced RPC beams;

$\alpha_{c} 、 \alpha_{s}-$ Carbon fiber reinforced coefficient and steel fiber reinforced coefficient; 
$\lambda_{c} 、 \lambda_{s}-$ The characteristic value of carbon fiber content and the characteristic value of steel fiber content, $\lambda_{c}=\rho_{c} l_{f c} / d_{f c}, \lambda_{s}=\rho_{s} l_{f s} / d_{f s}$;

Derivation of $\alpha_{c}$, take beam $\mathrm{C}_{0.5} \mathrm{R}$ for example.According to the physical properties of carbon fiber, the length of carbon fiber is $12 \mathrm{~mm}$, the diameter is $7 \mu \mathrm{m}, \lambda_{c}=8.5$. Bring $M_{u}=128.9 \mathrm{kN} \cdot \mathrm{m}, M_{c u}=130.6 \mathrm{kN} \cdot \mathrm{m}$ into formula (3), obtained $\alpha_{c}=0.00155$; Similarly, The beam $\mathrm{C}_{1.0} \mathrm{R}$ is obtained $\alpha_{c}=0.00154$; The beam $\mathrm{C}_{1.5} \mathrm{R}$ is obtained $\alpha_{c}=0.00166$; The beam $\mathrm{C}_{2.0} \mathrm{R}$ is obtained $\alpha_{c}=0.00042$, the average is $\alpha_{c}=0.0013$. Therefore, the ultimate bearing capacity of carbon fiber reinforced RPC beams is given by:

$$
M_{c u}=\left(1+0.0013 \lambda_{c}\right) M_{u}
$$

Derivation of $\alpha_{s}$, take beam $\mathrm{S}_{0.5} \mathrm{R}$ for example. According to the physical properties of steel fiber, the length of steel fiber is $13 \mathrm{~mm}$, the diameter is $0.2 \mathrm{~mm}, \lambda_{s}=0.325$, Bring $M_{u}=128.9 \mathrm{kN} \cdot \mathrm{m}, M_{c u}=131.5 \mathrm{kN} \cdot \mathrm{m}$ into formula (4), obtained $\alpha_{c}=0.0635$; Similarly, The beam $\mathrm{S}_{1.0} \mathrm{R}$ is obtained $\alpha_{c}=0.0629$, The beam $\mathrm{S}_{2.0} \mathrm{R}$ is obtained $\alpha_{c}=0.0448$, The beam $\mathrm{S}_{3.0} \mathrm{R}$ is obtained $\alpha_{c}=0.0269$, the average is $\alpha_{c}=0.0495$, Therefore, the ultimate bearing capacity of steel fiber reinforced RPC beams is given by:

$$
M_{\text {su }}=\left(1+0.0495 \lambda_{s}\right) M_{u}
$$

\section{Comparison of calculated values and simulated values}

Figure 10 is the scatter diagram of the calculated values and simulated values for the ultimate bearing capacity of fiber reinforced RPC beams.It can be seen from the figure, the calculated value is close to the simulated value, the relative error of both are within $5 \%$. Therefore, the formula of ultimate bearing capacity deduced in this paper is suitable for engineering calculation.
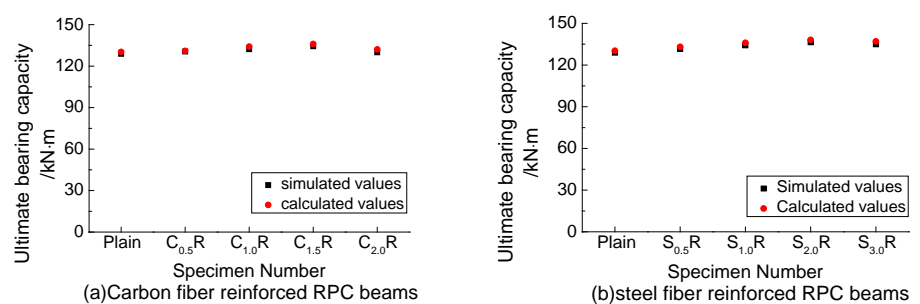

Fig. 10 Comparison between the calculated value and the simulated value of the ultimate bearing capacity

\section{Conclusion}

In this paper, the influence of different mixing ratio of carbon fiber and steel fiber on the bending moment and deflection of RPC beam in initial cracking state and yield state is analyzed by ANSYS finite element software under the environment prone to corrosion and ordinary environment. The following conclusions can be drawn:

(1) With the change of carbon fiber content, the flexural performance of RPC beam is improved. When the content of carbon fiber is $1.5 \%$, the initial crack is delayed effectively, and the mid-span bending moment of the beam is $60.4 \%$ higher than that of the Plain beam in the initial cracking state. After the crack is produced, the carbon fiber inhibits the development of the crack, and the toughness of the beam is improved. The mid-span bending moment of beam in yield state is $4.3 \%$ higher than that of Plain beam.

(2)With the change of steel fiber content, the flexural performance of RPC beam is also improved remarkably.In the initial cracking state, the mid-span bending moment of the beam is $142.3 \% \sim 163.3 \%$ higher than that of the Plain beam.In the yield state, the mid-span bending moment of the beam is $2.1 \% \sim 5.8 \%$ higher than that of the Plain beam. 
(3) Based on the calculation formula of flexural bearing capacity of RPC beams, considering the effect of fiber incorporation, the formula for flexural bearing capacity of fiber reinforced RPC beams is deduced, and the calculated values are in good agreement with the simulated values.

\section{References:}

[1]Cheyrezy M, Maret V, Frouin L. Microstructural analysis of RPC (Reactive Powder Concrete)[J]. Cement \& Concrete Research, 1995, 25(7):1491-1500.

[2]Yue-An L I. Study on Mechanical Properties of Polypropylene Fiber Reinforced Reactive Powder Concrete(RPC)[J]. China Concrete \& Cement Products, 2011.

[3]He K, Yang H, Jia F F, et al. Experimental Study on Mechanical Properties of Synthetic Macro-Fiber Reinforced Reactive Powder Concrete[J]. Applied Mechanics \& Materials, 2014, 496-500:2402-2406.

[4]Yang Z. Mechanical Properties of Steel Fiber and Polypropylene Fiber Reinforced Reactive Powder Concrete [J]. Industrial Construction, 2010, 40(9):77-79.

[5]Mingzhe An,Zihui Song,Li Yu, et al. Study on the Mechanical Properties of RPC Composites with Different Steel Fiber Content[J]. China Railway Science, 2009, 30(5):34-38.

[6]Jianlei Wang,Xiangyu Hao,Fengqiu Ji. Effect of Steel Fiber on Mechanical Properties of RPC Concrete[J]. Low Temperature Architecture Technology, 2008, 30(3):18-20.

[7]Zhaopeng Yang. Study on Mechanical Properties of Reactive Powder Concrete[D]. Dalian Jiaotong University, 2008.

[8]Zhaoning Wang.Research on Flexural Behavior of Reactive Powder Concrete Rectangular Beam with Steel Bars[D]. Beijing Jiaotong University,2008. 